Review

\title{
New Trends in the Therapeutic Approach to Metastatic Colorectal Cancer
}

\author{
Julia Sánchez-Gundín 1,2, Ana María Fernández-Carballido2,3, Lidia Martínez-Valdivieso1, Dolores \\ Barreda-Hernández ${ }^{1}$, Ana Isabel Torres-Suárez ${ }^{2,3}{ }^{凶}$
}

1. Hospital Pharmacist. Hospital Pharmacy Department. Virgen de la Luz Hospital, Hermandad de Donantes de Sangre, s/n, 16002 Cuenca (Spain).

2. Department of Pharmacy and Pharmaceutical Technology. Complutense University of Madrid, 28040 Madrid (Spain).

3. Institute of Industrial Pharmacy. Complutense University of Madrid, 28040 Madrid (Spain).

$\square$ Corresponding author: Ana Isabel Torres-Suárez. Department of Pharmacy and Pharmaceutical Technology. Complutense University of Madrid, 28040 Madrid (Spain). E-mail: galaaaa@ucm.es; Telephone number: (34) 913941735

(c) Ivyspring International Publisher. This is an open access article distributed under the terms of the Creative Commons Attribution (CC BY-NC) license (https:// creativecommons.org/licenses/by-nc/4.0/). See http://ivyspring.com/terms for full terms and conditions.

Received: 2017.12.19; Accepted: 2018.03.02; Published: 2018.04.03

\begin{abstract}
Important developments in chemotherapy for metastatic colorectal cancer over the last years are reviewed, with an emphasis on the most recently published data from clinical trials. The systematic review of current literature was conducted involving Pubmed Central ${ }^{\circledR}$ research and full articles were obtained and analyzed when appropriate.

Fluorouracil still constitutes the backbone of metastatic colorectal cancer treatment; fluorouracil combination plus either irinotecan (FOLFIRI), oxaliplatin (FOLFOX) or capecitabine (CAPOX or $X E L O X)$ are chemotherapy protocols established as treatments producing similar outcomes.

Actual treatment involves these chemotherapy protocols in combination with new molecular targeted drugs: bevacizumab and aflibercept (anti-vascular endothelial growth factor monoclonal antibody) and cetuximab and panitumumab (anti-epidermal growth factor receptor monoclonal antibody for patients with wild type KRAS) which confer significant survival benefits in select patients as first- or second-line therapies. The factors affecting the decisions for one treatment over other are related to the patient and toxicity drug.

Finally, metastatic colorectal cancer patients progressing after all standard therapies (maintaining a good ECOG performance status) could be candidates for further therapies such as regorafenib and TAS-102.

Regarding the future, promising therapies are under development for the metastatic colorectal cancer treatment and several agents are currently being evaluated in different clinical trials.
\end{abstract}

Key words: colorectal cancer, metastasis, targeted molecular therapies, chemotherapy

\section{Introduction}

Colorectal cancer (CRC) is one of the most common cancers worldwide with approximately $1,000,000$ people diagnosed annually [1]. CRC is the third most common cancer worldwide diagnosed for both genders, with a slightly higher incidence in males, after lung and breast cancer, and the fourth highest cause of cancer death after lung, liver and stomach cancer [2]. Approximately 25\% of patients present with metastases at initial diagnosis and almost $50 \%$ of patients with CRC will develop metastases, contributing to the high mortality rates reported for CRC [3].

5-fluorouracil (5-FU) has been the backbone of CRC treatment and it has been demonstrated that the use of combination regimens of 5-FU with cytotoxic agents, such as irinotecan and oxaliplatin, have improved the survival of patients with metastatic disease. Intravenous 5-FU can be substituted for capecitabine either as a single agent or combined with oxaliplatin, with the convenience of oral 
administration. All these regimens are generally considered interchangeable [4] and several variations of these combinations exist with the acronyms: FOLFOX (5-FU+oxaliplatin), XELOX or CAPOX (capecitabine+oxaliplatin) and FOLFIRI (5-FU+ irinotecan). The combination of capecitabine+ irinotecan (XELIRI) and 5-FU+oxaliplatin+irinotecan (FOLFOXIRI) are probably more toxic $[5,6,7]$.

This article reviews the clinical trial evidence for choosing optimal therapy in the treatment of patients with metastatic CRC (mCRC), incorporating new molecularly targeted drugs to chemotherapy agents.

\section{Metastatic colorectal cancer}

When tumour lesions become metastatic, the overall treatment strategy depends on whether the metastatic disease is respectable or only amenable to a palliative approach. The evaluation of resectability in patients with metastases should be standardized by a multidisciplinary committee, which should determinate, the goals of the treatment: prolongation of survival, cure, improving tumour-related symptoms, stopping tumour progression and/or maintaining quality of life.

\section{Resectable metastasic disease}

If the metastatic disease is resectable, the first-line treatment priority is immediate tumour control to ameliorate significant disease-related symptoms, to stabilize quickly progressing disease and to reduce metastases before surgery. The medical treatment of mCRC involves recently chemotherapy in combination with new molecular targeted drugs and these new regimens provide a higher resection rate (RR), longer progression free-survival (PFS) and better overall survival (OS) [8]. There is little difference in efficacy between FOLFOX and FOLFIRI and the main difference between these two combination therapies is the toxicity profile; more gastrointestinal side effects, fatigue and alopecia with FOLFIRI and more thrombocytopenia, neurotoxicity and hypersensitive reactions with FOLFOX $[1,9,10]$. Their combination with new molecular targeted drugs currently constitutes the backbone of systemic treatment of $\mathrm{mCRC}$. The choice of one regimen over the other depends on factors such as patient age, ECOG performance status, comorbidities, patient preferences and toxicity or drug availability. The exposure to all three cytotoxic agents (5-FU, oxaliplatin and irinotecan) in various sequences may result in a longest survival. Regarding to XELOX, it is an alternative to FOLFOX if it is used alone or in combination, with a more favourable side-effect profile (with the exception of a relatively higher rate of hand-and-foot syndrome) $[11,12,13,14]$.
The addition of bevacizumab (a recombinant humanized monoclonal anti-body (Mab) that binds to and neutralizes vascular endothelial growth factor (VEGF) [15]) to FOLFOX or XELOX first-line regimens resulted in a significant improved of PFS compared with placebo plus chemotherapy (Saltz et al. study [16]: 9.4 vs. 8.0 months; HR 0.83; $97.5 \%$ CI, 0.72 to 0.95 ; $\mathrm{p}=0.0023$ ). If bevacizumab is associated with FOLFIRI, Hurwitz et al. [17] demonstrated that the RR, PFS and OS were significantly higher in the group given FOLFIRI/bevacizumab than in the group given FOLFIRI/placebo: RR ( $44.8 \%$ vs. $34.8 \%$; $\mathrm{p}=0.004)$, PFS (10.6 vs. 6.2 months; HR 0.54; $\mathrm{p}<0.001)$ and OS (20.3 vs. 15.6 months; HR 0.66; $\mathrm{p}<0.001)$. Currently the regimens used in first-line treatment are FOLFOX, XELOX, or FOLFIRI plus bevacizumab and there are large observational studies that show no overall median PFS either OS differences between the different combination therapy regimens [17]. According to bevacizumab toxicity, it presents uncommon severe adverse events with an acceptable toxicity clinically manageable (hypertension, proteinuria, bleeding, thromboembolism, gastrointestinal perforation, wound healing) $[18,19]$.

When others treatments are desired, adding a Mab specifically directed against the epidermal growth factor receptor (EGFR) could be considered, either as a single agent or in combination with FOLFOX or FOLFIRI first-line regimens. Cetuximab and panitumumab are both EGFR-targeted Mab that block ligand-induced phosphorylation of EGFR, and signalling pathways involved in the control of cell survival, cell cycle progression, angiogenesis, cell migration and cellular invasion/metastasis [15]. These anti-EGFR Mab have demonstrated good efficacy and safety profile [20], but they are bit recommended in combination with capecitabinebased regimens.

Regarding genetic, it is known that KRAS gene mutation $(\mathrm{m} K R A S)$ is predictive of non-response to EGFR-targeted Mab therapy [21,22] but patients with mutations in codon 13 of the KRAS gene, which represent approximately $15-20 \%$ of all ${ }_{m}$ KRAS, seemed to respond to cetuximab with a similar magnitude to KRAS wild type $\left({ }_{w t} \mathrm{KRAS}\right)$ patients $[23,24]$. However, patients with mutations in exon 2 of KRAS are predictive of good outcomes for panitumumab treatment [25].

Bokemeyer et al. [26] conducted a phase II study of patients whose tumours were scored as ${ }_{w t} K R A S$. They compared FOLFOX/cetuximab vs. FOLFOX regimen, where the addition of cetuximab was associated with a higher RR $(61.0 \%$ vs. $37.0 \%$; OR 2.54 ; 95\% CI, 1.24 to 5.23; $\mathrm{p}=0.011$ ) and a longer PFS (7.7 vs. 7.2 months; HR 0.57; $95 \%$ CI, 0.36 to $0.90 ; \mathrm{p}=0.0163$ ). 
Moreover, the largest trial of addition of cetuximab to oxaliplatin-based chemotherapy concluded that cetuximab increases RR, with no evidence of benefit in PFS or OS in ${ }_{w t} K R A S$ [27].

Further, the efficacy of cetuximab plus FOLFIRI in the treatment of patients with mCRC with ${ }_{w t} \mathrm{KRAS}$ was evaluated by Van Cutsem et al. [28] where the addition of cetuximab to FOLFIRI significantly improved RR (57.3\% vs. 39.7\%; OD 2.07; 95\% CI, 1.51 to $2.80 ; \mathrm{p}=0.001$ ), PFS (9.9 vs. 8.4 months; HR 0.67; $95 \%$ $\mathrm{CI}, 0.56$ to $0.87 ; \mathrm{p}=0.0012$ ) and $\mathrm{OS}$ (23.5 vs. 20.0 months; HR 0.80; 95\% CI, 0.67 to 0.95; $\mathrm{p}=0.0093$ ) compared with FOLFIRI alone regimen. No significant difference in efficacy was evident in patients with ${ }_{m}$ KRAS.

Other study (Van Cutsem et al. study) [29] comparing FOLFIRI/cetuximab with FOLFIRI alone group provided confirmation that FOLFIRI/ cetuximab increased RR $(46.9 \%$ vs. $38.7 \%$; HR 1.40 ; 95\% CI, 1.12 to $1.77 ; \mathrm{p}=0.004)$ and PFS (8.9 vs. 8.0 months; HR 0.85; 95\% CI, 0.72 to 0.99 ; $\mathrm{p}=0.048$ ). However, there was no significant difference between the treatment groups in OS.

The most common adverse event at any grade related to cetuximab was diarrhea and skin/ subcutaneous tissue disorders [26].

Heinemann et al. [30] recruited patients in order to compare the association of FOLFIRI/cetuximab with FOLFIRI/ bevacizumab in first line patients with ${ }_{\mathrm{w}} \mathrm{K}$ KRAS. Although the proportion of patients who achieved an objective response and the PFS were not significantly different between both groups (objective response: $62.0 \%$ vs. $58.0 \%$; OR $1.18 ; 95 \% \mathrm{CI}, 0.85$ to 1.64, $\mathrm{p}=0.18$ and PFS: 10.0 vs. 10.3 months; HR 1.06; $95 \% \mathrm{CI}, 0.88$ to $1.26, \mathrm{p}=0.55$ ), this study suggests that FOLFIRI/cetuximab could be the preferred first line schedule because of its association with a significant longer OS (28.7 vs. 25.0 months; HR 0.77; 95\% CI, 0.62 to $0.96 ; \mathrm{p}=0.017)$. Safety profiles were according to known side effect.

Because of that, all chemotherapy (FOLFOX/FOLFIRI) plus cetuximab or bevacizumab combinations should be regarded as appropriate.

As far as panitumumab is concerned, the PRIME trial [31] compared FOLFOX/panitumumab vs. FOLFOX in previously untreated ${ }_{\mathrm{w}} \mathrm{KR}$ KSS patients. A statically significant improvement in PFS was observed compared with FOLFOX alone regimen (9.6 vs. 8.0 months; HR 0.80; $95 \% \mathrm{CI}, 0.66$ to 0.97 ; $\mathrm{p}=0.02$ ), but did not lead to a significant improvement in median OS (23.9 vs. 19.7 months; HR 0.83; 95\% CI, 0.67 to $1.02 ; \mathrm{p}=0.072)$. Complete resections after metastasectomy of any site were achieved in $8.3 \%$ of patients treated with FOLFOX/panitumumab and $7.0 \%$ of patients treated with FOLFOX. The most common adverse event related to panitumumab was skin toxicity.

In the PEAK study [32], FOLFOX/panitumumab was also compared with FOLFOX/bevacizumab regimen in patients with previously untreated ${ }_{\text {wt }}$ KRAS mCRC resulting in similar PFS (10.9 vs. 10.1 months; HR 0.87; 95\% CI, 0.65 to 1.17; $\mathrm{p}=0.353)$, but longer OS favouring panitumumab arm (34.2 vs. 24.3 months; HR $0.87 \%$; $95 \%$ CI, 0.44 to 0.89 ; $\mathrm{p}=0.009$ ).

Regarding the addition of an anti-EGFR Mab to standard first-line chemotherapy/bevacizumab, two phase III trials showed a shorter PFS, major toxicity and inferior quality of life compared with the standard therapy [33,34].

Different chemotherapy regimens in first-line in the mCRC treatment are summarized in table 1.

Table 1. Mainly clinical trial and targeted therapies in first-line metastatic colorectal treatment.

\begin{tabular}{|c|c|c|c|c|c|c|c|}
\hline Study & Design & RR & p-value & Median PFS & $p$-value & Median OS & p-value \\
\hline \multirow[t]{3}{*}{ Saltz et al. [16] } & Bevacizumab + FOLFOX/XELOX & - & & 9.4 & & - & \\
\hline & vs. & & - & & 0.002 & & - \\
\hline & FOLFOX/XELOX & - & & 8 & & - & \\
\hline \multirow[t]{3}{*}{ Hurwitz et al. [17] } & Bevacizumab + FOLFIRI & 44.8 & & 10.6 & & 20.3 & \\
\hline & vs. & & 0.004 & & $<0.001$ & & $<0.001$ \\
\hline & FOLFIRI & 34.8 & & 6.2 & & 15.6 & \\
\hline \multirow[t]{3}{*}{ Bokemeyer et al. [26] } & Cetuximab + FOLFOX & 61 & & 7.7 & & - & \\
\hline & vs. & & 0.011 & & 0.0163 & & - \\
\hline & FOLFOX & 37 & & 7.2 & & - & \\
\hline \multirow[t]{3}{*}{ Van Cutsem et al. [28] } & Cetuximab + FOLFIRI & 57.3 & & 9.9 & & 23.5 & \\
\hline & vs. & & 0.001 & & 0.0012 & & 0.0093 \\
\hline & FOLFIRI & 39.7 & & 8.4 & & 20.0 & \\
\hline \multirow[t]{3}{*}{ Van Cutsem et al. [29] } & Cetuximab + FOLFIRI & 46.9 & & 8.9 & & 19.9 & \\
\hline & vs. & & 0.004 & & 0.048 & & 0.31 \\
\hline & FOLFIRI & 38.7 & & 8.0 & & 18.6 & \\
\hline \multirow[t]{3}{*}{ PRIME study [31] } & Panitumumab + FOLFOX & - & & 9.6 & & 23.9 & \\
\hline & vs. & & - & & 0.02 & & 0.072 \\
\hline & FOLFOX & - & & 8.0 & & 19.7 & \\
\hline
\end{tabular}

RR: response rate (\%). PFS: progression free-survival (months). OS: overall survival (months). 
Even though the increases of RR, PFS and OS, many patients inevitably relapsed due to the newly acquired tumor resistance or have a tumor progression that involve switching to salvage therapies in second, and subsequent lines of chemotherapy treatment. For most patients progression following an oxaliplatin doublet will result in a switch to irinotecan-based therapy and vice-versa.

Respect to the choice of Mab in second-line therapy, it depends as in the first-line treatment on various factors: KRAS status, patient age, ECOG performance status and comorbidities, toxicity and drug availability.

In the second-line setting, the Eastern Cooperative Oncology Group 3200 study [35] enrolled patients previously treated with FOLFIRI and found that RR $(22.7 \%$ vs. $8.6 \%$; p<0.0001), PFS (7.3 vs. 4.7 months; HR 0.61; $\mathrm{p}<0.0001)$ and OS (12.9 vs. 10.8 months, HR 0.75; $p=0.0011$ ) were all significantly improved with FOLFOX/bevacizumab treatment compared with FOLFOX alone. This association were related to an increase of toxicities (14\%) grade 3-4 such as hypertension, bleeding, nausea vomiting. According to FOLFIRI, the addition of bevacizumab to this regimen was associated with a trend of OS improved (25.1 vs. 22.2 months) [17].

For patients with ${ }_{w t}$ KRAS, EGFR-targeting Mabs provide an additional option for second-line or subsequent salvage therapies because have clear benefits in later lines of therapy.

The EPIC trial [36] aimed to determine whether the addition of cetuximab to FOLFIRI prolonged survival in patients previously treated with oxaliplatin-based therapy. The results showed higher RR ( $16.4 \%$ vs. $4.2 \%$; $\mathrm{p}=0.0001)$, longer PFS (4.0 vs. 2.6 months; HR $0.69,95 \% \mathrm{CI}, 0.62$ to $0.78 ; \mathrm{p}=0.0001)$ and similar OS (10.7 vs. 10.0 months; HR 0.97, 95\% CI, 0.85 to $1.11 ; \mathrm{p}=0.71)$ in cetuximab arm. These data were also observed in the ELSIE study [37] where patients from Asia and Australia were involved.

Regarding to panitumumab, Peeters et al. [38] presented its combination with FOLFIRI in second-line treatment, which resulted in a higher median PFS (5.9 vs. 3.9 months; HR 0.73; 95\% CI, 0.59 to $0.90 ; \mathrm{p}=0.004)$ and a not statically significant increase in median OS (14.5 vs. 12.5 months; HR 0.85, $95 \% \mathrm{CI}, \quad 0.70$ to $1.04 ; \mathrm{p}=0.12$ ) in favour of panitumumab subpopulation.

Nevertheless, published data comparing the addition of cetuximab or panitumumab to FOLFOX in second-line treatment with FOLFOX therapy alone are very limited and to emphasize that triple combination of chemotherapy + anti-EGFR Mab + anti-VEGF Mab therapy is not recommended for the treatment of patients with mCRC.

Others therapies are aflibercept and ramucirumab, a recombinant fusion protein and Mab whose mechanism of action is to target VEGF and placental growth factor by blocking angiogenesis [15]. It has only been demonstrated to have activity when they are used in combination with FOLFIRI in oxaliplatin pre-treated patients. Respect to aflibercept, this recombinant fusion protein has to be used in oxaliplatin pre-treated patients regardless of whether they have been pre-treated with bevacizumab in first-line therapy. The results were confirmed in the VELOUR study [39] where the addition of aflibercept to FOLFIRI showed a better results on the aflibercept arm: higher RR (19.8\% vs. $11.1 \%$; $\mathrm{p}=0.0001)$, PFS increase (6.90 vs. 4.67 months; HR 0.76; $95 \%$ CI, 0.66 to $0.87 ; \mathrm{p}=0.0001)$ and median OS improvement (13.50 vs. 12.06 months; HR 0.82 ; $95.34 \%$ CI, 0.71 to 0.94 ; $\mathrm{p}=0.0032$ ). The security of this combination resulted in an enhancing of adverse effects associated with FOLFIRI plus aflibercept treatment-related toxicities (hypertension, mucosal bleeding, proteinuria) [39]. According to ramucirumab, this Mab has to be used in oxaliplatin and bevacizumab pre-treated patients. RAISE [40] study showed an improvement in ramucirumab plus FOLFIRI treated patients comparing to placebo plus FOLFIRI patients (PFS: 5.7 vs. 4.5 months; HR 0.793; IC 95\%: 0.697 to 0.903; $\mathrm{p}=0.0005$; and OS: 13.3 vs. 11.7 months; HR 0.844; IC 95\%: 0.730 to $0.976 ; p=0.0219$ ). Grade 3 or worse adverse events seen in more than $5 \%$ of patients were neutropenia (38\%), with febrile neutropenia incidence of $3 \%$, hypertension (11\%), diarrhoea $(11 \%)$ and fatigue $(12 \%)$, with a treatment-related deaths similar in both study arms (2.4\% ramucirumab plus FOLFIRI vs. $2.0 \%$ placebo plus FOLFIRI) [41].

Different chemotherapy regimens in no first-line mCRC treatment are summarized in table 2.

\section{Progression after standard therapies}

Many patients progressing after all standard therapies maintain a good ECOG performance status and adequate organ function. They could be candidates for further therapy such as regorafenib and TAS-102, whose activity is not affected by KRAS status.

These further therapies have the advantage of oral administration. Regorafenib is an oral tumour deactivation agent that potently blocks multiple protein kinases, including kinases involved in tumour angiogenesis and oncogenesis [15]. In the CORRECT trial [42], regorafenib treatment resulted in a statistically significant improvement compared to placebo in PFS (2.0 vs. 1.7 months; HR 0.49; 95\% CI, 0.42 to $0.58 ; p=0.0001]$ and in an OS prolongation (6.4 
vs. 5.0 months; HR 0.77 ; $95 \% \mathrm{CI}, 0.64$ to 0.94 ; $\mathrm{p}=0.0102]$. Moreover, $93 \%$ of patients experienced any treatment adverse events and the most frequent were fatigue and hand-foot-skin reaction.

Table 2. Mainly clinical trial and targeted therapies in no first-line metastasic colorectal treatment.

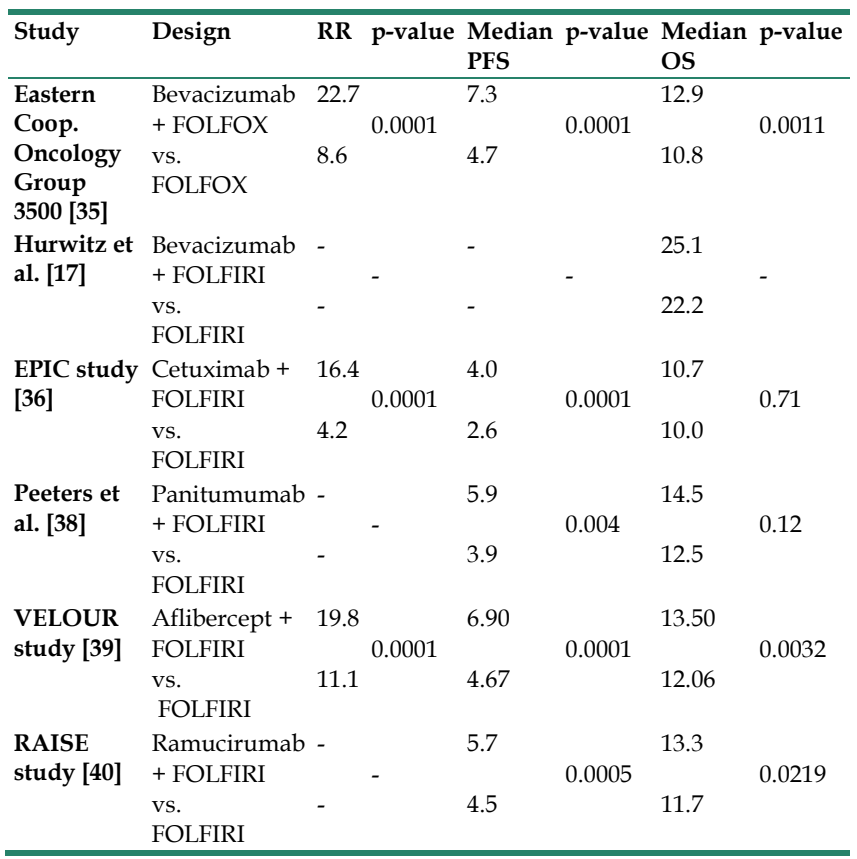

RR: response rate (\%). PFS: progression free-survival (months). OS: overall survival (months).

A second phase III, international, multi-center, randomized, double blind, placebo-controlled study (CONCUR) is another study where regorafenib efficacy and safety are evaluated. Results of CONCUR [43] study involved a significant longer PFS (3.2 vs. 1.7 months; HR 0.55, 95\% CI, 0.40 to 0.77 ; $\mathrm{p}=0.00016$ ) and OS (8.8 vs. 6.3 months; HR $0.31,95 \%$ CI, 0.22 to 0.94 ; $\mathrm{p}>0.001)$ than in CORRECT trial. This difference could be explained because of a less heavily pre-treated patient population in CONCUR study. Therefore, data from this study suggest that regorafenib should be used before patients deteriorate and before the reuse of previous lines of chemotherapy. It is important to ensure that patients receive regorafenib when they still have a good ECOG performance status and are suitable candidates. According to safety, 97\% of the patients in CONCUR study had an adverse event during regorafenib treatment and the most frequent were hand-foot-skin reaction, hypertension, hyperbilirubinemia, hypophosphataemia and alanine aminotransferase concentration increases.

TAS-102, it is another novel oral agent. It is an anti-tumour agent composed of the cytotoxin trifluridine and the thymidine phosphorylase inhibitor tipiracil that prevents the degradation of trifluridine, maintaining an effective blood concentration of trifluridine. In the phase III RECOURSE trial [44], TAS-102 was associated with a significantly prolongation of PFS comparing to placebo (2.0 vs. 1.7 months; HR 0.48; 95\% CI, 0.41 to 0.57 ; $\mathrm{p}<0.001$ ) and OS (7.1 vs. 5.3 months; HR 0.68; 95\% CI, 0.58 to 0.81 ; $\mathrm{p}<0.001)$. In particular, the efficacy of TAS-102 was documented in patients with disease that had been refractory to 5-FU when that drug had been administered as a component of the last treatment regimen before study entry and in patients who had previously treatment with regorafenib. The addition of TAS-102 to best supportive care, as compared with placebo plus best supportive care, resulted in a significant delay in the worsening of ECOG performance status from the baseline of 0 or 1 to 2 or higher (the median time to worsening in ECOG performance status was 5.7 months with TAS-102 compared with 4 months for placebo (HR, 0.66)). Neutropenia was the main reported adverse event, occurring in $38 \%$ of patients treated with TAS- 102 . Overall, febrile neutropenia occurred in $4 \%$ of patients, with $9 \%$ receiving granulocyte colony-stimulating factor as treatment. In conclusion, TAS-102 is considered well tolerated with mild toxic effects [45] in no more than $10 \%$ of patients.

Unfortunately, there is no pre-treatment biomarker that indicates which patients will or will not benefit from regorafenib and TAS-102 treatment and nowadays, the most important selection criteria for these treatments are patient's clinical factors (age, ECOG performance status, comorbilities) and history of previous treatment. Clinical experience shows that patients who have a deteriorated ECOG performance status do not respond to these treatments because are likely to experience adverse events without any benefit.

\section{Palliative treatment}

Finally, most patients with mCRC present un-resectable disease and will be only candidates for palliative treatment. For such patients, chemotherapy plus bevacizumab or chemotherapy alone would be an appropriate first-line treatment choice depending on organ function and comorbidities. Notwithstanding the addition of EGFR-targeted Mab therapy to combination chemotherapy in ${ }_{w t}$ KRAS patients could also be considered in this setting. The aims of palliative therapy are prolongation of survival, symptom control and maintenance of quality of life. Classical determinants affecting the choice of systemic treatment can be divided into those relating to the patient (age, comorbidities and preferences), those relating to the disease (tumour resectability, disease burden, symptoms, rate of disease progression and 
prior treatment history) and those relating to the drug (toxicity, availability and cost).

For patients who cannot tolerate combination chemotherapy, or with slowly progressing and/or non-symptomatic disease, a potentially low toxicity chemotherapy regimen, such as 5-FU monotherapy, could enable the addition of a single biological agent more safely.

\section{Metastatic Colorectal Cancer Future}

Regarding the future, promising therapies are under development for the mCRC treatment. Several agents are currently being evaluated in different clinical trials including $[46,47]$ :

- Chemotherapy agents: topotecan (topoisomerase I inhibitor).

- Targeted therapies: trastuzumab (targeting overexpressed human epidermal growth factor receptor [HER] 2), erlotinib (targeting overexpressed HER1), lapatinib (dual HER1/2 inhibitor), onartuzumab (hepatocyte growth factor inhibitor), mapatumumab (tumour necrosis factor-related apoptosis-inducing ligand receptor-1 inhibitor), astivantinib, brivanib, cediranib and cabozantinib (tyrosine kinase inhibitor), vismodegib, dasatinib, saracatinib, bosutinib.

- Immunotherapies: nivolumab (anti-programmed death-1 receptor Mab), ipilimumab (anti-cytotoxic T-lymphocyte antigen-4 Mab).

\section{Conclusions}

Substantial improvements have been made in the management of CRC over the last two decades. Treatment for mCRC is based largely on the stage of the cancer, but other factors, including any previous treatments and overall health, can also be important in order to decide the optimal treatment strategy. This strategy should be discussed by a multidisciplinary expert team.

The outcome of patients diagnosed with mCRC has clearly improved during recent years from an OS approximately of 12 months to nearly 30 months in recent clinical trials [48]. This improvement has been because of the integration of new cytotoxic agents and targeted therapies and these more effective therapeutic combinations have increased furthermore the rate of curative-intent surgical resections.

\section{Acknowledgments}

This work has been prepared in collaboration between the Complutense University of Madrid, Spain, and the Virgen de la Luz Hospital, Cuenca, Spain.

\section{Competing Interests}

The authors have declared that no competing interest exists.

\section{References}

1. Choong MK, Tsafnat G. Genetic and Epigenetic Biomarkers of Colorectal Cancer. Clin Gastroenterol Hepatol. 2012; 10(1): 9-15.

2. Ferlay J, Soerjomataram I, Dikshit R, Eser S, Mathers C, Rebelo M et al. Cancer incidence and mortality worldwide: sources, methods and major patterns in GLOBOCAN 2012. Int J Cancer. 2015; 136(5): E359-86.

3. Van Cutsem E, Cervantes A, Nordlinger B, Arnold D. Metastatic colorectal cancer: ESMO Clinical Practice Guidelines for diagnosis, treatment and follow-up. Ann Oncol. 2014; 25(3): 1-9.

4. Vera R, Alonso V, Gállego J, González E, Guillín-Ponce C, Pericay C et al. Current controversies in the management of metastasic colorectal cancer. Cancer Chemother Pharmacol. 2015; 76(4): 659-77.

5. Montagnani F, Chiriatti A, Turrisi G, Francini G, Fiorentini G. A systematic review of FOLFOXIRI chemotherapy for the first-line treatment of metastatic colorectal cancer: improved efficacy at the cost of increased toxicity. Colorectal Dis. $2011 ; 13(8)$ : 846-52

6. Cersosimo RJ. Management of advanced colorectal cancer, Part 1. Am J Health Syst Pharm. 2013; 70(5): 395-406.

7. Cersosimo RJ. Management of advanced colorectal cancer, Part 2. Am J Health Syst Pharm. 2013; 70(6): 491-506.

8. Ribecco AS, Pino MS, Cipriani G, Marinozzi C, Fioretto L. Molecularly targeted therapy: toxicity and quality of life considerations in advanced colorectal cancer. Expert Rev Anticancer Ther. 2013; 13(10): 1181-91.

9. Colucci G, Gebbia V, Paoletti G, Giuliani F, Caruso M, Gebbia N et al. Phase III randomized trial of FOLFIRI versus FOLFOX4 in the treatment of advanced colorectal cancer: a multicenter study of the Gruppo Oncologico Dell'Italia Meridionale. J Clin Oncol. 2005; 23(22): 4866-75.

10. Tournigand C, André T, Achille E, Lledo G, Flesh M, Mery-Mignard D et al. FOLFIRI followed by FOLFOX6 or the reverse sequence in advanced colorectal cancer: a randomized GERCOR study. J Clin Oncol. 2004; 22(2): 229-37.

11. Díaz-Rubio E, Tabernero J, Gómez-España A, Massutí B, Sastre J, Chaves M et al. Phase III study of capecitabine plus oxaliplatin compared with continuous-infusion fluorouracil plus oxaliplatin as first-line therapy in metastatic colorectal cancer: final report of the Spanish Cooperative Group for the Treatment of Digestive Tumors Trial. J Clin Oncol. 2007; 25(27): 4224-30.

12. Antonuzzo L, Giommoni E, Pastorelli D, Latiano T, Pavese I, Azzarello D et al. Bevacizumab plus XELOX as first-line treatment of metastatic colorectal cancer: The OBELIX study. World J Gastroenterol. 2015; 21(23): 7281-8.

13. Cassidy J, Clarke S, Díaz-Rubio E, Scheithauer W, Figer A, Wong R et al. Randomized phase III study of capecitabine plus oxaliplatin compared with fluorouracil/folinic acid plus oxaliplatin as first-line therapy for metastatic colorectal cancer. J Clin Oncol. 2008; 26(12): 2006-12.

14. Guo Y, Xiong BH, Zhang T, Cheng Y, Ma L. XELOX vs. FOLFOX in metastatic colorectal cancer: An updated meta-analysis. Cancer Invest. 2016; 34(2): 94-104.

15. [Internet] Agencia Española de Medicamentos y Productos Sanitarios. Summary of product charactheristics. Revised 25 May 2016. http://www.aemps.gob.es/cima/fichasTecnicas.do?metodo=buscar

16. Saltz LB, Clarke S, Díaz-Rubio E, Scheithauer W, Figer A, Wong R et al. Bevacizumab in combination with oxaliplatin-based chemotherapy as first-line therapy in metastatic colorectal cancer: a randomized phase III study. J Clin Oncol. 2008; 26(12): 2013-9.

17. Hurwitz H, Fehrenbacher L, Novotny W, Cartwright T, Hainsworth J, Heim W. Bevacizumab plus irinotecan, fluorouracil, and leucovorin for metastatic colorectal cancer. N Engl J Med. 2004; 350(23): 2335-42.

18. Puthillath A, Patel A, Fakih MG. Targeted therapies in the management of colorectal carcinoma: role of bevacizumab. Onco Targets Ther. 2009; 2: 1-15.

19. Qu CY, Zheng Y, Zhou M, Zhang Y, Shen F, Cao J et al. Value of bevacizumab in treatment of colorectal cancer: A meta-analysis. World J Gastroenterol. 2015; 21(16): 5072-80.

20. Mello RA, Marques AM, Araújo A. Epidermal growth factor receptor and metastatic colorectal cancer: insights into target therapies. World J Gastroenterol. 2013; 19(38): 6315-8.

21. Qi-Bin Song, Qi Wang, Wei-Guo Hu. Anti-epidermal growth factor receptor monoclonal antibodies in metastatic colorectal cancer: A meta-analysis. World J Gastroenterol. 2015; 21(14): 4365-72.

22. Sorich MJ, Wiese MD, Rowland A, Kichenadasse G, McKinnon RA, Karapetis CS. Extended RAS mutations and anti-EGFR monoclonal antibody survival benefit in metastatic colorectal cancer: a meta-analysis of randomized, controlled trials. Ann Oncol. 2015; 26(1): 13-21.

23. Tejpar S, Celik I, Schlichting M, Sartorius U, Bokemeyer C, Cutsem EV. Association of KRAS G13D tumor mutations with outcome in patients with metastatic colorectal cancer treated with first-line chemotherapy with or without cetuximab. J Clin Oncol. 2012; 30(29): 3570-77. 
24. Van Cutsem E, Lenz HJ, Köhne CH, Heinemann V, Tejpar S, Melezínek I et al. Fluorouracil, leucovorin, and irinotecan plus cetuximab treatment and RAS mutations in colorectal cancer. J Clin Oncol. 2015; 33(7): 692-700.

25. Douillard JY, Oliner KS, Siena S, Tabernero J, Burkes R, Barugel M et al. Panitumumab-FOLFOX4 Treatment and RAS Mutations in Colorectal Cancer. N Engl J Med. 2013; 369(11): 1023-34.

26. Bokemeyer C, Bondarenko I, Makhson A, Hartmann JT, Aparicio J, Braud F et al. Fluorouracil, Leucovorin, and Oxaliplatin With and Without Cetuximab in the First-Line Treatment of Metastatic Colorectal Cancer. J Clin Oncol. 2009: 27(5): 663-71.

27. Maughan TS, Adams RA, Smith CG, Meade AM, Seymour MT, Wilson RH et al. Addition of cetuximab to oxaliplatin-based first-line combination chemotherapy for treatment of advanced colorectal cancer: Results of the randomised phase 3 MRC COIN trial. Lancet. 2011; 377(9783): 2103-14.

28. Van Cutsem E, Köhne CH, Lang I, Folprecht G, Nowacki MP, Cascinu S et al. Cetuximab Plus Irinotecan, Fluorouracil, and Leucovorin As First-Line Treatment for Metastatic Colorectal Cancer: Updated Analysis of Overall Survival According to Tumor KRAS and BRAF Mutation Status. J Clin Oncol. 2011; 29(15): 2011-19.

29. Van Cutsem E, Köhne CH, Hitre E, Zaluski J, Chang Chien CR, Makhson A et al. Cetuximab and chemotherapy as initial treatment for metastatic colorectal cancer. N Engl J Med. 2009; 360(14): 1408-17.

30. Heinemann V, von Weikersthal LF, Decker T, Kiani A, Vehling-Kaiser U, Al-Batran SE et al. FOLFIRI plus cetuximab versus FOLFIRI plus bevacizumab as first-line treatment for patients with metastatic colorectal cancer (FIRE-3): a randomised, open-label, phase 3 trial. Lancet Oncol. 2014; 15(10): 1065-75.

31. Douillard JY, Siena S, Cassidy J, Tabernero J, Burkes R, Barugel M et al. Randomized, Phase III Trial of Panitumumab With Infusional Fluorouracil, Leucovorin, and Oxaliplatin (FOLFOX4) Versus FOLFOX4 Alone As First-Line Treatment in Patients With Previously Untreated Metastatic Colorectal Cancer: The PRIME Study. J Clin Oncol. 2010; 28(31): 4697-705.

32. Schwartzberg LS, Rivera F, Karthaus M, Fasola G, Canon JL, Hecht JR et al. PEAK: a randomized, multicenter phase II study of panitumumab plus modified fluorouracil, leucovorin, and oxaliplatin (mFOLFOX6) or bevacizumab plus mFOLFOX6 in patients with previously untreated, unresectable, wild-type KRAS exon 2 metastatic colorectal cancer. J Clin Oncol. 2014; 32(21): 2240-7.

33. Hecht JR, Mitchell E, Chidiac T, Scroggin C, Hagenstad C, Spigel D et al. A Randomized Phase IIIB Trial of Chemotherapy, Bevacizumab, and Panitumumab Compared With Chemotherapy and Bevacizumab Alone for Metastatic Colorectal Cancer. J Clin Oncol. 2009; 27(5): 672-80.

34. Tol J, Koopman M, Cats A, Rodenburg CJ, Creemers GJM, Schrama JG et al. Chemotherapy, Bevacizumab, and Cetuximab in Metastatic Colorectal Cancer. N Engl J Med. 2009; 360(6): 563-72.

35. Giantonio BJ, Catalano PJ, Meropol NJ, O'Dwyer PJ, Mitchell EP, Alberts SR et al. Bevacizumab in combination with oxaliplatin, fluorouracil, and leucovorin (FOLFOX4) for previously treated metastatic colorectal cancer: results from the Eastern Cooperative Oncology Group Study E3200. J Clin Oncol. 2007; 25(12): 1539-44.

36. Sobrero AF, Maurel J, Fehrenbacher L, Scheithauer W, Abubakr YA, Lutz MP et al. EPIC: Phase III trial of cetuximab plus irinotecan after fluoropyrimidine and oxaliplatin failure in patients with metastatic colorectal cancer. J Clin Oncol. 2008; 26(14): 2311-9.

37. Lim R, Sun Y, Im SA, Hsieh RK, Yau TK, Bonaventura A et al. Cetuximab plus irinotecan in pretreated metastatic colorectal cancer patients: The ELSIE study. World J Gastroenterol. 2011; 17(14): 1879-88.

38. Peeters M, Price TJ, Cervantes A, Sobrero AF, Ducreux M, Hotko Y et al. Randomized phase III study of panitumumab with fluorouracil, leucovorin, and irinotecan (FOLFIRI) compared with FOLFIRI alone as second-line treatment in patients with metastatic colorectal cancer. J Clin Oncol. 2010; 28(31): 4706-13.

39. Van Cutsem E, Tabernero J, Lakomy R, Prenens H, Prausová J, Macarulla T et al. Addition of aflibercept to fluorouracil, leucovorin, and irinotecan improves survival in a phase III randomized trial in patients with metastatic colorectal cancer previously treated with an oxaliplatin-based regimen. J Clin Oncol. 2012; 30(28): 3499-506

40. Tabernero J, Yoshino T, Cohn AL, Obermannova R, Bodoky G, Garcia-Carbonero $R$ et al. Ramucirumab versus placebo in combination with second-line FOLFIRI in patients with metastatic colorectal carcinoma that progressed during or after first-line therapy with bevacizumab, oxaliplatin, and a fluoropyrimidine (RAISE): a randomised, double-blind, multicentre, phase 3 study. Lancet Oncol. 2015; 16(5): 499-508.

41. Diaz-Serrano A, Riesco-Martinez MC, Garcia-Carbonero R. The safety and efficacy of ramucirumab for the treatment of metastatic colorectal cancer. Expert Rev Anticancer Ther. 2016; 16(6): 585-95.

42. Grothey A, Van Cutsem E, Sobrero A, Siena S, Falcone A, Ychou M et al. Regorafenib monotherapy for previously treated metastatic colorectal cancer (CORRECT): An international, multicentre, randomised, placebo-controlled, phase 3 trial. Lancet. 2013; 381(9863): 303-12.

43. Li J, Qin S, Xu R, Yau TC, Ma B, Pan H et al. Regorafenib plus best supportive care versus placebo plus best supportive care in Asian patients with previously treated metastatic colorectal cancer (CONCUR): a randomised, double-blind, placebo-controlled, phase 3 trial. Lancet Oncol. 2015; 16(6): 619-29.
44. Mayer RJ, Cutsem EV, Falcone A, Yoshino T, Garcia-Carbonero R, Mizunuma $\mathrm{N}$ et al. Randomized Trial of TAS-102 for Refractory Metastatic Colorectal Cancer. N Engl J Med. 2015; 372(20): 1909-19.

45. Yoshino T, Mizunuma N, Yamazaki K, Nishina T, Komatsu Y, Baba H et al. TAS-102 monotherapy for pretreated metastatic colorectal cancer: a double-blind, randomised, placebo-controlled phase 2 trial. Lancet Oncol. 2012; 13(10): 993-1001.

46. Sun $W$. Angiogenesis in metastatic colorectal cancer and the benefits of targeted therapy. J Hematol Oncol. 2012; 5: 63.

47. Shukla RK. Recent advances in oral anticancer agents for colon cancer. Future Oncol. 2013; 9(12): 1893-908.

48. Fakih MG. Metastatic colorectal cancer: current state and future directions. Clin Oncol. 2015; 33(16): 1809-24. 